\title{
Markdown Time for Perishables Based on Dynamic Quality Evaluation for Complex Data Analysis
}

\author{
Jiang-Tao Wang, ${ }^{1}$ Jian-Jun Yu, ${ }^{2}$ Yu-Hsi Yuan $\mathbb{D}^{3}{ }^{3}$ Sang-Bing Tsai $\mathbb{D},{ }^{4}$ and Shu-Fen Zhang $\mathbb{D}^{5}$ \\ ${ }^{1}$ Zhongshan Institute, University of Electronic Science and Technology of China, Zhongshan 528400, China \\ ${ }^{2}$ School of Business Administration, South China University of Technology, Guangzhou 510641, China \\ ${ }^{3}$ Department of Labor \& Human Resources, Chinese Culture University, Taipei 111, Taiwan \\ ${ }^{4}$ Regional Green Economy Development Research Center, School of Business, WUYI University, Wuyishan 354300, China \\ ${ }^{5}$ School of Chinese Medicine, Guangdong Pharmaceutical University, Guangzhou 510006, China \\ Correspondence should be addressed to Yu-Hsi Yuan; yuanyh@gm.ypu.edu.tw and Shu-Fen Zhang; zsf063@gdpu.edu.cn
}

Received 9 December 2020; Revised 28 December 2020; Accepted 31 December 2020; Published 3 February 2021

Academic Editor: Yuanpeng Zhang

Copyright (c) 2021 Jiang-Tao Wang et al. This is an open access article distributed under the Creative Commons Attribution License, which permits unrestricted use, distribution, and reproduction in any medium, provided the original work is properly cited.

\begin{abstract}
Perishables' freshness is a dynamic state, which can be captured by statistical big data based on advanced product identification and sensory technologies. A time-based markdown strategy model is proposed to consider the markdown strategy in maximizing performance. The results showed that demand uncertainty would force them to implement a markdown strategy in advance, merchants' profit and initial inventory are sensitive to the uncertainty and deterioration rate of perishable quality, and markdown time has a regulatory effect on initial inventory.
\end{abstract}

\section{Introduction}

Freshness is becoming a quality criterion of great importance to consumers regarding perishable products (Peneau et al. [1]). The freshness as well as the price has been viewed as an important factor influencing consumers' purchase decision. Many companies are making efforts to promote the fresh concept in the retail market. Perishable operation management must consider how to deal with perishables before they spoil to ensure food safety. Sales at discounted prices have become a common strategy in retail processes, especially with long lead times and hard-to-predict demand. As a common method of matching supply and demand, the markdown strategy has a dramatic effect on a retailer's profitability [2], i.e., too early or too deeply will lead to lost revenue, while delaying markdowns or keeping them shallow will lead to liquidating inventory at even lower prices at the end of the season [3]. Hence, effective markdown strategies should not ignore the timing of the markdown as well as the initial inventory.
Perishable's typical characteristic is that the freshness (i.e., quality) is a dynamic state that decreases continuously to the point when it is unfit for consumption. Most advanced product identification and sensory technologies such as radio frequency identification technology (RFID) and time temperature indicator (TTI) have been developed to make it possible to capture the perishables' quality information, such as product identity, properties, and related data [48]. These rich quality index data can be used to predict dynamically the shelf life. At present, some research literature of perishable products focus on the preservation technology investment [9-11] and some research considered the price and inventory problem of perishables by considering the markdown time as an exogenous variable [12-14]. However, how to effectively implement markdown strategies consisting of time and inventory based on the dynamic shelf life information should be an important issue in perishable operation management, especially in an uncertain demand environment. 
A time-sensitive markdown strategy is employed to consider the markdown time challenge based on the timevariance market sale model. This time-variance markdown model can capture the dynamic quality evolution characteristics. The relations between the markdown time and the initial inventory are considered under uncertainty market demand. Partial decision-making attributes are demonstrated through numerical analysis. The results suggest that market uncertainty may not always be detrimental to merchants and may even increase the ultimate benefit to them. Both the merchant's profit and initial inventory are sensitive to the market demand uncertainty while the markdown time is not.

The rest of this paper is organized as follows. Section 2 presents a review of the existing literature related to our research. The model description is presented in Section 3. We will consider the markdown strategy under deterministic and uncertain market demand, respectively, in Sections 4 and 5. Section 6 presents numerical results based on the theoretical results. A summary of this research and discussion of future research directions are conducted in Section 7. Some proofs are presented in supplementary files (available here).

\section{Literature Review}

Perishables' production and inventory control problems have been given more attention in the inventory literature $[12,15]$. In the study of perishables, the research relating to this study can be grouped into two streams.

A stream of related research literature focuses on the preservation technology investment. These researches consider how to control and reduce the deterioration of perishables through effective procedural changes and specialized equipment acquisition. The main theme is to decide the investment in preservation technologies to reduce deterioration rates using product inventory models [9-11]. Fox example, Shah et al. [16] derived the optimal preservation technology investment, retail price, and purchase quantity for inventory systems where units are subject to constant rates of deterioration.

Another stream of related research literature focuses on the price and inventory control model, which either considers the product with fixed lifetimes, random lifetimes, and decay rates that correspond to proportional inventory decreases in terms of utility or quantity $[14,17,18]$; or focuses on combining pricing and inventory controls to investigate how different pricing modalities affect pricing and inventory decisions [19-22]; or considers how to adjust prices to attract customers over one or more periods in a certain demand market $[13,23]$. These researches suggested that customer valuations have significant effects on retailers' price and inventory decisions [24-26], and markdown strategy in operation management is a controversial topic. In particular, the markdowns would not be viewed as an optimal pricing modality for monopoly firms when the firm has high levels of inventory [27], and has often been characterized as a wasteful practice [28]. However, markdowns have also been suggested to be a more profitable strategy than single fixed prices [29] and a better pricing modality when customer behavior is considered $[30,31]$. We noticed that these researches always viewed the markdown time as an exogenous variable when considering price and/or inventory for perishables. However, this paper differs in that it views the markdown time as a decision variable and considers it under uncertainty market demand.

Demand uncertainty is an important issue in operation management literature. Perishables' market demand is also uncertain and would be influenced by the dynamic quality evolution. The newsvendor model is always used to consider the decision problem under uncertain demand [32, 33]. However, most of the extant newsvendor model literature assumed that demand uncertainty is exogenous. The timevariant market demand model in a quick response system assumed that lead-time reduction can help to enhance forecast accuracy of uncertain market demand [34-36] and was then employed to consider the lead-time decisions (purchase timing, guaranteed delivery time, lead time, and options contracts) in the supply chain [36-39]. Inspired by this stream of research, we assumed the perishables' demand uncertainty is time-sensitive and relates to the sale period length, which is also common in reality, i.e., the longer the sale period, the greater the uncertainty. The literature that is closely related to this study is [3] and that by Wang et al. [40]. However, Şen [3] considered the markdown time for perishable and substitutable produce under a determined market, and Wang et al. [40] consider the introduction time for a holiday product with similar cumulative market demand model. The main difference from the above literature is that this study assumed that the market demand rate is price- and time-sensitive, then considers the markdown time as well as the initial inventory strategy under uncertain markets.

\section{Model Description}

All perishables are available for a limited sale period and must be sold before the "sell-by-date," which has been printed on product labels by the producer to assure the product is of satisfactory quality. The perishables' freshness then can be represented in terms of remaining shelf life. The effects of remaining shelf life on freshness with the market demand are expressed commonly by the kinetic model approach in the literature [41]. An exponential quality-decay weighting function (i.e., $\beta q e^{-\lambda t}$ ) derived from the kinetic model approach has been widely adopted in perishables' product research $[14,42]$ then a price and quality sensitive expected market demand rate for perishable product can be viewed as $f(D(t))=f(p(t), t)=d-\alpha p(t)+\beta q(t)=d-\alpha p(t)+\beta q e^{-\lambda t}$, where $d(>0)$ is the maximum potential market demand rate, $\lambda$ represents the quality deterioration rate, and $\alpha, \beta(\geq 0)$ represent demand sensitivity to the product price and product quality, respectively. The market demand rate satisfies $d f$ $(D(t)) / d p \leq 0, d f(D(t)) / d t \leq 0$. The expected market demand during the selling season $[0, T]$ is then $D(T)=$ $\int_{0}^{T} f(p(t), t) d t$.

Merchants prepare inventory in advance and then appropriately use markdown pricing during the season to reverse the declining demand. Theoretically, dynamic pricing strategy $p(t)$ can maintain the expected demand rate at a high 
level as the quality deteriorates. However, dynamic pricing methods incur change cost, and too many markdowns represent failure in some area [43]. A single price change may be as effective as the more flexible pricing ([44]; Sen, 2013). Hence, it is reasonable to assume that merchants would have only one chance to implement the markdown strategy during the selling season. If the merchant makes the markdown price at time $z \in(0, T)$, then the retail price would be expressed as $p(t)=\{p, 0 \leq t \leq z ; p \theta, z<t \leq T, \theta \in(0,1]\}$ with a price discount $\theta$, and then the expected market sales are divided into two parts as $E\left(D_{1}(p(t), t)\right)=\int_{0}^{z} f(p(t), t) d t=$ $\mu_{0 z}(p(z), z), E\left(D_{2}(p(t), t)\right)=\int_{z}^{T} f(p(t), t) d t=\mu_{z T}(p(z), z)$.

The errors of the actual market sales relate to the sale time $[35,40]$. So, market sale can be expressed as $D_{1}(p(t)$, $t)=\mu_{0 z}+\varepsilon(z), D_{2}(p(t), t)=\mu_{z T}+\varepsilon(T-z)$, where $\varepsilon(t)$ is the stochastic part of market sales and satisfies $d \operatorname{Var}(\varepsilon(t)) / d t$ $\leq 0$ (Chiu, Choi, and Li 2009). Following Chen and Chuang [35] and Wang et al. [40], we assume $\varepsilon(t)=((T-t) / T) \sigma X$, where $X \sim N(0,1)$ with the pdf $f(x)$ and cdf $F(x)$ and its inverse function $F^{-1}(x)$. A larger $\sigma$ means higher market demand uncertainty. $\operatorname{ED}(p(t), t)$ must be larger than $\sigma$ so that $\operatorname{Pr}(D(p(t), t) \leq 0$ can be negligible.

Merchant's markdown strategies are to consider the initial inventory $(Q)$ and markdown time $z$ to improve the operation performance with the quality evolving over time. In order to focus on the initial inventory and the markdown time decisions under uncertain market demand, the initial price $p$ and the markdown price are exogenous and predetermined as assumed in [3]. Then, the merchant's optimal decision function is

$$
\pi(p(t), z)=\sum_{i=1}^{2} p(t) \min \left(Q, D_{i}(t)\right)-c Q .
$$

\section{The Markdown Strategy under Deterministic Market Demand}

As a benchmark, we first consider markdown strategy under the deterministic market demand. Then, the actual market demand is $\operatorname{ED}(p, \theta, z)=\int_{0}^{z} d-a p+\beta q e^{-\lambda t} d t+\int_{z}^{T} d-a p \theta+\beta$ $q e^{-\lambda t} d t=(\beta q / \lambda)\left(1-e^{-\lambda T}\right)+d T-a p(\theta T+(1-\theta) z)$ with the predetermined price $p(t)=\{p, 0 \leq t \leq z ; p \theta, z<t \leq T, \theta \in(0$, $1]\}$, and the profit function can be viewed as $\pi(z, Q)=p \int_{0}^{z}$ $d-a p+\beta q e^{-\lambda t} d t+p \theta \int_{z}^{T} d-a p \theta+\beta q e^{-\lambda t} d t-c Q$ with $Q=E$ $D(p, \theta, z)$.

Theorem 1. Under deterministic market demand, there exists unique optimal markdown time for the merchant's expected profit function as $z^{*}=(1 / \lambda) \ln (\beta q /(a(p(1+\theta)-c)-d))$ and then the initial inventory is $Q=(\beta q / \lambda)\left(1-e^{-\lambda T}\right)+d T$ $-a p\left(\theta T+(1-\theta) z^{*}\right)$.

Proof. Direct derivation of the profit function is sufficient.

Proposition 2. The higher the initial retail price, the earlier the markdown time; the higher the discount price, the later the markdown time; and the later the markdown time, the smaller the inventory would be.

Proof. Direct derivation is sufficient as $\partial z^{*} / \partial p \leq 0, \partial z^{*} / \partial \theta \leq 0$

Theorem 1 and Proposition 2 suggest that if a merchant sets a high initial price, then he will implement the price reduction early. However, if the merchant plans to take a significant price cut, he would delay the price reduction time. Eventually, the willingness to delay price reduction would lead to conservative initial inventory.

\section{The Markdown Strategies under Uncertain Market Demand}

Under an uncertain market demand, the merchant can adopt the markdown strategy under two scenarios as follows.

5.1. Inventory Clearance Markdown. If the markdown price is aimed at clearing the inventory, then it can be viewed as the salvage price in most operation literature, i.e., $p \theta \leq c$. Then, the merchants' profit function is $\pi(Q, z)$ $=E\left(p \min (Q, D)+p \theta(Q-D)^{+}\right)-c Q=p \min \left(\mu_{0 z}(p(z))+\right.$ $\varepsilon(z), Q)+p \theta\left(Q-\mu_{0 z}(p(z))-\varepsilon(z)\right)^{+}-c Q=(p-c) Q-p(1-$ $\theta)(T-z / T) \sigma \int_{-\infty}^{\left(\left(Q-\mu_{0 z}\right) / \sigma\right)(T /(T-z))} F(x) d x$.

Lemma 3. For any markdown time $z$, the merchant's profit function is a concave in the initial inventory.

Proof. Derive the profit function twice on $Q$ and then obtain that $\partial \pi(Q, t) / \partial Q=(p-c)-p(1-\theta) \cdot F(((Q-\mu) / \sigma)(T /(T-$ $z))), \partial^{2} \pi(Q, t) / \partial Q^{2}=-(p(1-\theta) / \sigma)(T / T-z) \cdot f(((Q-\mu) /$ $\sigma)(T /(T-z))) \leq 0$.

Theorem 4. To clear the inventory with the markdown price, the merchant would delay the markdown time to the expiration rate or time as the demand rate decreases to zero, i.e., $z^{*}=T$ or $z^{*}=(1 / \lambda) \ln (\beta q /(a p-d))$, and the inventory is $Q^{*}=(d-a p) z^{*}+\left(1-e^{-\lambda z^{*}}\right) \beta q / \lambda+\sigma\left(T-z^{*}\right) / T$. $F^{-1}((p-c) / p(1-\theta))$.

Proposition 5. If the markdown price is aimed at clearing the inventory, the markdown time may be earlier than that under the deterministic market demand.

Proof. Compare the markdown times as follows, $a(p(1+$ $\theta)-c)=a p+a(p \theta-c) \leq a p(p \theta \leq c)$, then $(1 / \lambda) \ln (\beta q /(a p$ $-d)) \leq(1 / \lambda) \ln (\beta q /(a(p(1+\theta)-c)-d)) \leq T$.

5.2. Promotional Markdown. If the markdown price is not to clear the leftover inventory at once but to reverse the declining demand caused by quality deterioration, it would occur whenever the demand rate cannot reach the expected value. Then, the markdown price would not surely clear the inventory. The market sales before and after the markdown price can be viewed as $\min \left(Q, D_{1}\right)$ and $\min \left(Q-\min \left(Q, D_{1}\right)\right.$, 


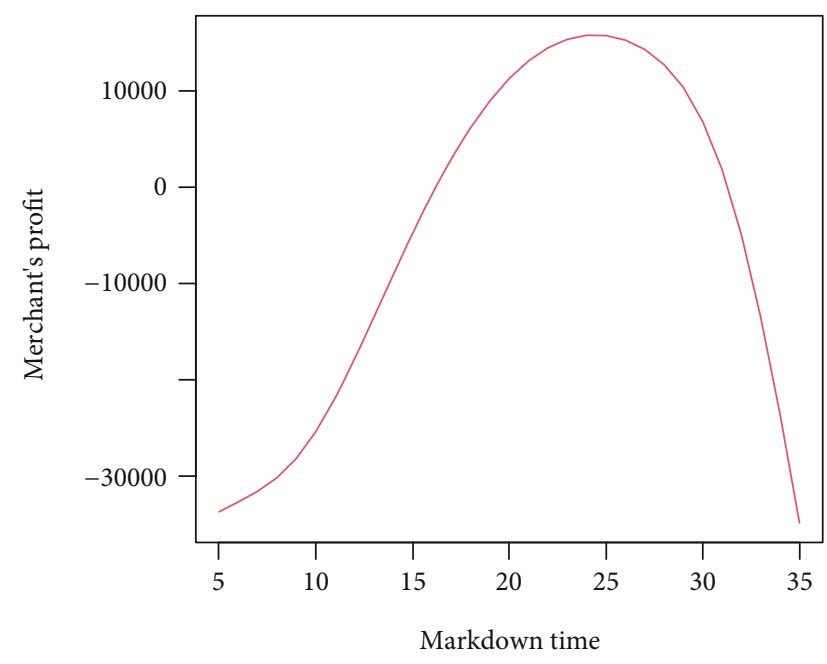

(a)

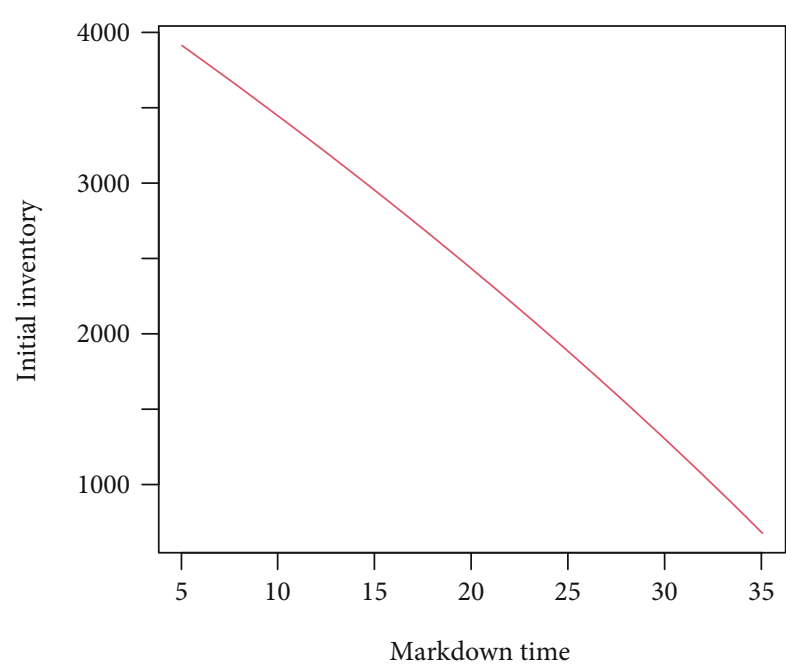

(b)

Figure 1: Merchant's profit (a) and initial inventory (b) on the markdown time.

$\left.D_{2}\right)$. The merchant's profit function can be expressed as $\pi(Q, p)=\mathbf{E}\left(p \min \left(Q, D_{1}\right)+p \theta \min \left(Q-\min \left(Q, D_{1}\right), D_{2}\right)\right)$ $-c Q$. Drawing on the approach used by Netessine and Shumsky (2005) and noting that $\min \left(Q, D_{1}\right)=\{Q,(1 / \sigma)(T /$ $\left.(T-z))\left(Q-\mu_{0 z}\right) \leq x ; D_{1},(1 / \sigma)(T /(T-z))\left(Q-\mu_{0 z}\right) \geq x\right\}$ and $\min \left(Q-\min \left(Q, D_{1}\right), D_{2}\right)=\{0,(1 / \sigma)(T /(T-z))(Q-$ $\left.\mu_{0 z}\right) \leq x ; Q-D_{1},\left(Q-\mu_{0 T}\right) / \sigma \leq x \leq(1 / \sigma)(T /(T-z))(Q-$ $\left.\left.\mu_{0 z}\right) ; D_{2}, x \leq\left(Q-\mu_{0 T}\right) / \sigma\right\}$, the merchant's profit function can be rewritten as $\pi(Q, p)=p\left(\int_{-\infty}^{(T / z)\left(\left(Q-\mu_{1}\right) / \sigma\right)}\left(\mu_{0 z}+(z / T) \sigma\right.\right.$ $\left.x) f(x) d x+\int_{(T / z)\left(\left(Q-\mu_{0 z}\right) / \sigma\right)}^{\infty} Q f(x) d x\right)+p \theta\left(\int_{\left(Q-\left(\mu_{0 z}+\mu_{z T}\right)\right) / \sigma}^{(T / z)\left(\left(Q-\mu_{0}\right) / \sigma\right)}(Q-\right.$ $\left.\mu_{0 z}-(z / T) \sigma x\right) f(x) d x+\int_{-\infty}^{\left(Q-\left(\mu_{0 z}+\mu_{z T}\right)\right) / \sigma}\left(\mu_{z T}+((T-z) / T) \sigma x\right) f$ $(x) d x)-c Q=(p-c) Q-p(1-\theta)(z / T) \sigma \int_{-\infty}^{(T / z)\left(\left(Q-\mu_{0 z}\right) / \sigma\right)} F(x) d$ $x-p \theta \sigma \int_{-\infty}^{\left(Q-\left(\mu_{0 z}+\mu_{z T}\right)\right) / \sigma} F(x) d x$.

Lemma 6. For any markdown time $z$, the merchant's profit function $\pi(Q, z)$ is a concave in the initial inventory $Q$. The optimal initial inventory $Q^{*}(z)$ satisfies the equation as $p-$ $c-p(1-\theta) F\left((T / z)\left(\left(Q-\mu_{0 z}\right) / \sigma\right)\right)-p \theta F\left(\left(Q-\left(\mu_{0 z}+\mu_{z T}\right)\right) /\right.$ $\sigma)=0$.

Lemma 7. The initial inventory satisfies $(z / T) \sigma F^{-1}((p-c)$ / $p)+\mu_{0 z} \leq Q^{*} \leq \sigma F^{-1}((p-c) / p)+\mu_{z T}+\mu_{0 z}$, i.e.,

(1) if $(c / p) \leq 0.5$, then $F^{-1}((p-c) / p) \geq 0$, and $0 \leq(z / T)$ $\sigma F^{-1}((p-c) / p)+\mu_{0 z} \leq Q^{*} \leq \sigma F^{-1}((p-c) / p)+\mu_{z T}$

(2) if $(c / p) \geq 0.5$, then $F^{-1}((p-c) / p) \leq 0$, then $\sigma(\geq 0)$ satisfies that

(a) if $\sigma F^{-1}((p-c) / p)+\mu_{2} \geq 0$, then $\mu_{0 z} \leq Q^{*} \leq \sigma F^{-1}((p$ $-c) / p)+\mu_{0 z}+\mu_{z T}$

(b) if $\sigma F^{-1}((p-c) / p)+\mu_{2} \leq 0$, then $Q^{*} \leq \mu_{0 z}$
Theorem 8. There exists an optimal markdown time $z^{*}$, which can be found by the following algorithm:

(1) For any given $z$, solving the equation $p-c-p(1-\theta) F$ $\left((T /(T-z))\left(\left(Q-\mu_{0 z}\right) / \sigma\right)\right)-p \theta F\left(\left(Q-\mu_{0 T}\right) / \sigma\right)=0$ to obtain the optimal inventory $Q^{*}$; (2) substituting the $Q^{*}$ into the profit function and then obtaining the $\pi\left(Q^{*}, z\right)$; (3) computing value $\pi\left(Q^{*}, z\right)$ on $z \in(0, T)$ and then obtaining the optimal $z^{*}=$ $\arg \max \pi\left(Q^{*}, z\right)$.

Proof. Substituting the $Q^{*}(z)$ into the merchant's profit function and then obtaining $\pi\left(Q^{*}(z), z\right)$. Note that $\pi\left(Q^{*}(z), z\right)$ is a nonlinear univariate continuous function and satisfies $\lim _{z \rightarrow 0^{+}} \pi\left(Q^{*}(z), z\right)=\pi\left(Q^{*}(0), 0\right)$ and $\lim _{z \rightarrow T^{-}} \pi\left(Q^{*}(z), z\right)=(p-c)$ $Q-p \theta \sigma \int_{-\infty}^{\left(Q-\mu_{0 \rightarrow T}\right) / \sigma} F(x) d x$. Actually, if $z \rightarrow 0^{+}$, the markdown strategy degenerates into a daily low price strategy. So, there must exist an optimal time $z^{*} \in[0, T]$ that satisfies $z^{*}=\arg$ $\max \pi\left(Q^{*}(z), z\right)$.

Lemma 7 and Theorem 8 suggest that there exists an optimal markdown time for a single price change strategy. The initial inventory is influenced by the initial price and the uncertainty in market sales. Tiny margins and large uncertainty would seriously dampen the merchant's initial inventory. Markdown strategy with time decisions can provide different insights for perishable operation management. Although the optimal initial inventory and markdown time can be derived, more decisions and the relations among the decision variables cannot be obtained analytically due to the nonlinearity of expressions. Therefore, more market insights implied in these complex relationship structures need to be explored by numerical analysis.

\section{Numerical Analysis}

Before performing the numerical analysis, we need to reorganize the constraints among parametric variables such as that the $\operatorname{ED}(p(t), t)$ must be larger than $\sigma$ so that $\operatorname{Pr}(D(p(t), t)$ 

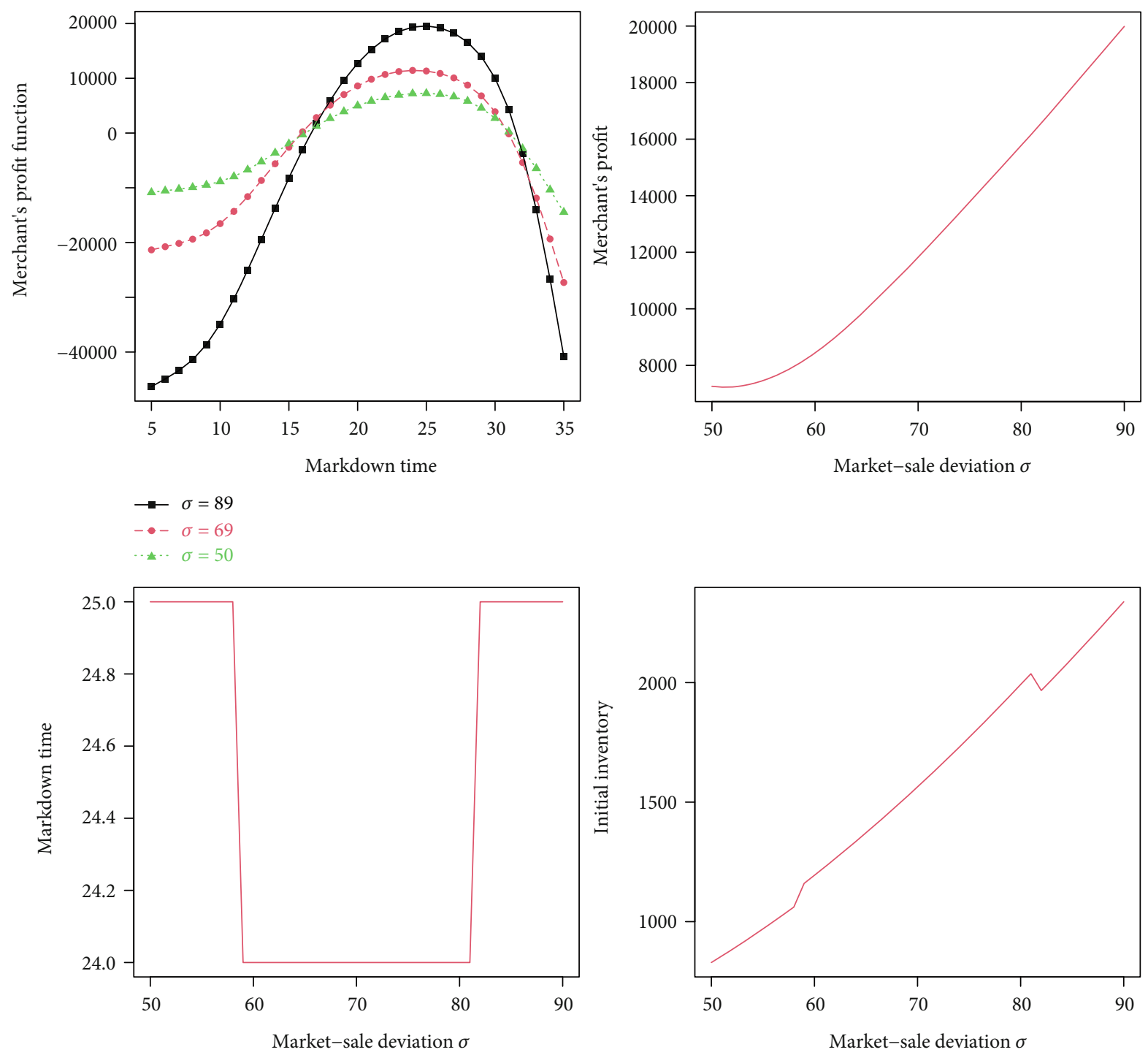

Figure 2: The market-sale deviation on the decisions.

$\leq 0$ can be negligible, and the parameters $\alpha, \beta$ must satisfy that $0 \leq a p-d \leq a(p(t)(1+\theta)-c)-d \leq \beta q e^{-\lambda t}, t \in(0, T)$. It is reasonable to assume the values of some parameters as market size $d=70$; initial quality $q=10$; demand sensitivity to price and quality $a=1$ and $\beta=1.5$, respectively; quality deterioration $\lambda=0.02$; selling period length $T=40$; and product $\operatorname{cost} c=22$, initial price $p=80$, and $\theta=0.25$; the stochastic part of market sales is assumed to follow the normal distribution, i.e., $x \in N(0,80)$.

6.1. The Revenue and Initial Inventory on Markdown Time. In order to demonstrate the effectiveness and feasibility of the algorithm in Theorem 8, Figure 1 presents the initial inventory and merchant's revenue with the markdown time evolution. Consistent with the theoretical analysis, the merchant's revenue function is a concave function on the markdown time. Hence, there exists an optimal markdown time. Besides, although the optimal initial inventory satisfies a special equation, the relationship between the initial inventory and markdown time can be approximately linear.
6.2. The Effect of Uncertainty on the Markdown Strategies. Based on the algorithm in Theorem 8 , the merchant's profit function, finial profit, markdown time, and initial inventory with different market-sale deviation are presented in Figure 2. Figure 2 suggests that the merchant's profit as well as the initial inventory increases with the market-sale deviation increase. Theorem 1 points out that the merchant would delay the markdown time under a deterministic demand, which will result in small initial inventory and finally reduce the sale volumes as well as the profit during the sale period. However, uncertainty would prompt merchants to implement early price reduction, which ultimately increases the potential market sale volumes during the sale season. The markdown time does not seem to be very sensitive to the deviation, but indeed affects the extent of the initial inventory.

6.3. The Effect of Quality Deterioration Rate on the Markdown Strategies. The most valued characteristic of perishables is the perishability rate. The higher the perishability 

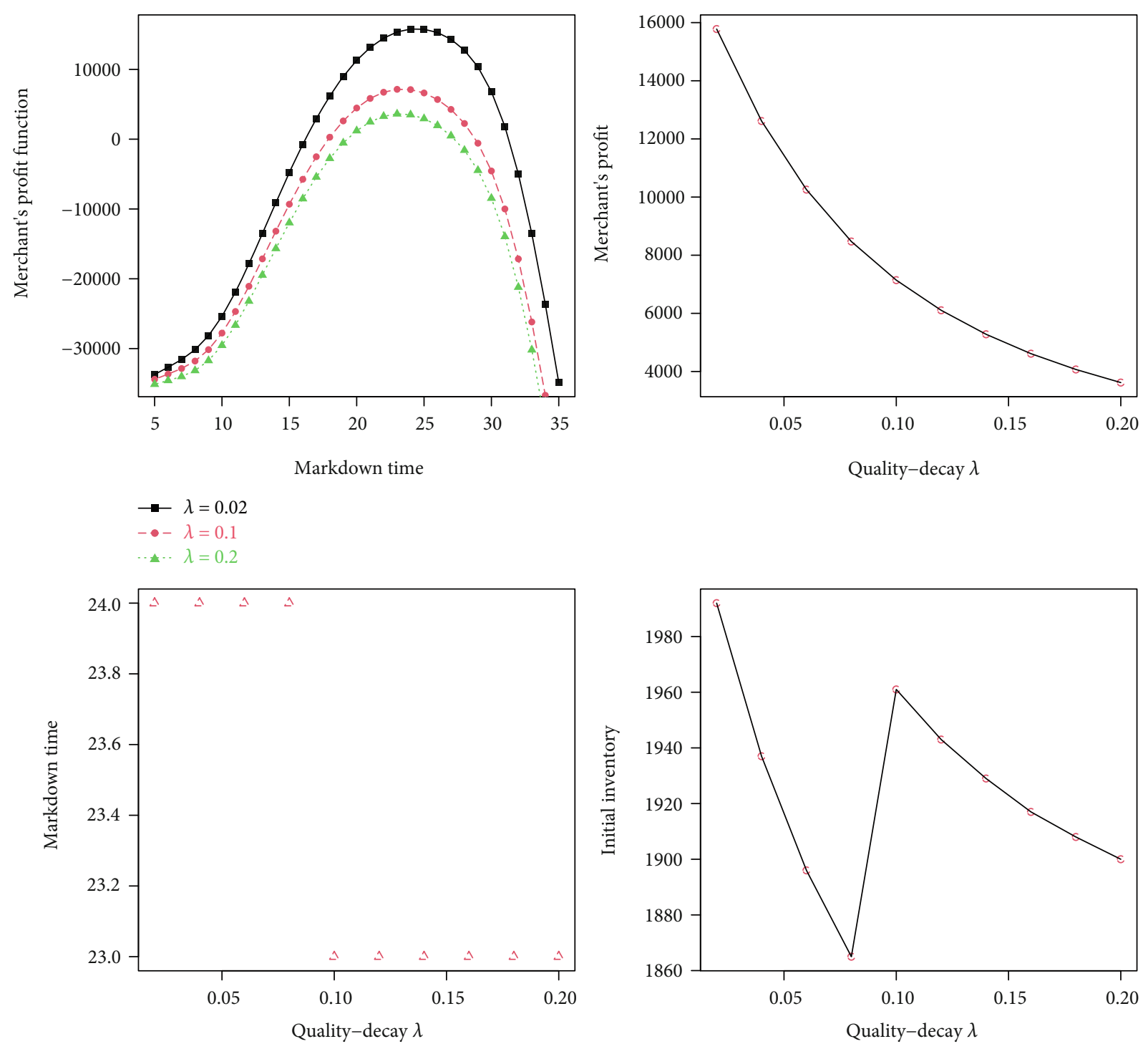

FIgURE 3: Effects' deterioration rate on markdown times.

rate, the more difficult it is to preserve. Figure 3 suggests that the merchant's profit decreases with the deterioration rate increase. So, the more perishable the product, the less profitable the merchant gets. The markdown time also does not seem too sensitive to the deterioration rate. It plays a role in regulating the change of the initial inventory to the deterioration rate. Generally, the shorter the shelf life of the product, the more difficult it is for the merchant to handle it, and the lower the final profit would be. In reality, some merchants operating in perishables always adopt high initial prices to quickly cover costs. In particular, products that are too perishable are even sold directly in presales or a custom model.

\section{Conclusion}

Freshness and price have become major issues in the management of perishables with the increasing attention paid to the freshness of perishables. The markdown strategy is a common operating method to reverse the declining demand due to the dynamic quality evaluation. An effective markdown strategy can avoid waste due to the expiration dates when optimizing operational performance. Statistical big data based on advanced product identification and sensory technologies can capture perishables' freshness state and model the evolution of the freshness of perishables with shelf life (such as $\beta q e^{-\lambda t}$ ) [45-49]. So, the time-sensitive markdown strategies are feasible. By introducing a time-variant market demand model, we consider the relationship between the optimal markdown time and the optimal initial inventory under uncertain market demand. The optimal markdown time as well as the initial inventory is derived under different scenarios. The results show that there exists an optimal markdown time under various scenarios; the uncertainty of market sales would force merchants to implement a markdown strategy in advance. The early implementation of the markdown strategy would then stimulate the potential market demand, which finally increases the merchant's profit. Hence, both the profit and initial inventory increase with 
the uncertain market increase and decrease with the increase in the quality deterioration rate. The dynamic deterioration of perishable quality harms merchant's revenue. The more perishable the product, the less the merchant is willing to prepare in advance. Therefore, many perishables are sold in a customized way or presale in reality. Besides, markdown time is less sensitive to the uncertainty and perishability but has a regulatory effect on initial inventory.

Markdown time as an important issue in markdown strategy can provide new and significant insights for merchants. This research on time-sensitive markdown strategies enriches current markdown strategy research and provides a theoretical basis for an intelligent decision-making system for perishable product management. However, it should be noted that the markdown strategy in this study only focuses on the initial inventory and markdown time under uncertain market demand. It is undeniable that the initial price and the magnitude of the markdown price are also important factors in markdown strategies. Hence, future research on markdown strategies should include more decision variables to provide more insights into market strategies.

\section{Data Availability}

The data used to support the findings of this study are available from the corresponding authors upon request.

\section{Conflicts of Interest}

The authors have declared that no competing interests exist concerning this study.

\section{Acknowledgments}

The work was supported by the Provincial Nature Science Foundation of Guangdong (2018A030313488, 2016A060311539), Guangdong Province Young Innovative Talents Program in Universities (Natural Science) (No. 2018KQNCX327), Guangdong Province Philosophy and Social Sciences "13th Five-Year" Planning Project for Building Discipline (GD17XGL56), Guangdong Soft Science Research Project (2018A070712005), and Fundamental Research Funds for the Central Universities (2018MSXM01).

\section{Supplementary Materials}

The proofs of some theorems and properties are presented in the supplementary materials. (Supplementary Materials)

\section{References}

[1] S. Péneau, P. B. Brockhoff, F. Escher, and J. Nuessli, “A comprehensive approach to evaluate the freshness of strawberries and carrots," Postharvest Biology and Technology, vol. 45, no. 1, pp. 20-29, 2007.

[2] J. Zhang, G. Liu, Q. Zhang, and Z. Bai, "Coordinating a supply chain for deteriorating items with a revenue sharing and cooperative investment contract," Omega, vol. 56, no. 3, pp. 37-49, 2015.
[3] A. Şen, "Competitive markdown timing for perishable and substitutable products," Omega, vol. 64, pp. 24-41, 2016.

[4] Y. Jiang, X. Gu, D. Wu et al., "A novel negative-transferresistant fuzzy clustering model with a shared cross-domain transfer latent space and its application to brain CT image segmentation," IEEE/ACM Transactions on Computational Biology and Bioinformatics, vol. 99, pp. 1-13, 2019.

[5] Y. Jiang, Y. Zhang, C. Lin, D. Wu, and C. T. Lin, "EEG-based driver drowsiness estimation using an online multi-view and transfer TSK fuzzy system," IEEE Transactions on Intelligent Transportation Systems, vol. 99, pp. 1-13, 2020.

[6] Y. Zhang, F. L. Chung, and S. Wang, "Clustering by transmission learning from data density to label manifold with statistical diffusion," Knowledge-Based Systems, vol. 193, p. 105330, 2019.

[7] Y. Zhang, S. Wang, K. Xia, Y. Jiang, and P. Qian, “Alzheimer's disease multiclass diagnosis via multimodal neuroimaging embedding feature selection and fusion," Information Fusion, vol. 66, pp. 170-183, 2021.

[8] Y. Zhang, J. Li, X. Zhou, T. Zhou, and J. Yang, "A viewreduction based multi-view TSK fuzzy system and its application for textile color classification," Journal of Ambient Intelligence and Humanized Computing, vol. 1, pp. 1-11, 2019.

[9] T. Hsieh and C. Dye, "A production-inventory model incorporating the effect of preservation technology investment when demand is fluctuating with time," Journal of Computational and Applied Mathematics, vol. 239, no. 2, pp. 25-36, 2013.

[10] P. H. Hsu, H. M. Wee, and H. M. Teng, "Preservation technology investment for deteriorating inventory," International Journal of Production Economics, vol. 124, no. 2, pp. 388394, 2010.

[11] Y. P. Lee and C. Dye, "An inventory model for deteriorating items under stock-dependent demand and controllable deterioration rate," Computers and Industrial Engineering, vol. 63, no. 2, pp. 474-482, 2012.

[12] W. Elmaghraby and P. Keskinocak, "Dynamic pricing in the presence of inventory considerations: research overview, current practices, and future directions," Management Science, vol. 49, no. 10, pp. 1287-1309, 2003.

[13] I. Hassouneh, T. Serra, and Š. Bojnec, "Nonlinearities in the Slovenian apple price transmission," British Food Journal, vol. 117, no. 1, pp. 461-478, 2015.

[14] X. Wang and D. Li, "A dynamic product quality evaluation based pricing model for perishable food supply chains," Omega, vol. 40, no. 6, pp. 906-917, 2012.

[15] I. Z. A. S. Karaesmen, Managing Perishable and Aging Inventories: Review and Future Research Directions, K. G. A. K. Kempf, Ed., Springer US, New York, NY, 2011.

[16] N. H. Shah, D. B. Shah, and D. G. Patel, "Optimal preservation technology investment, retail price and ordering policies for deteriorating items under trended demand and two level trade credit financing," Journal of Mathematical Modelling \& Algorithms in Operations Research, vol. 14, no. 1, pp. 1-12, 2015.

[17] S. K. Goyal and B. C. Giri, "Recent trends in modeling of deteriorating inventory," European Journal of Operational Research, vol. 134, no. 1, pp. 1-16, 2001.

[18] F. Raafat, "Survey of literature on continuously deteriorating inventory models," The Journal of the Operational Research Society, vol. 42, no. 1, pp. 27-37, 1991. 
[19] P. L. Abad, "Optimal pricing and lot-sizing under conditions of perishability and partial backordering," Management Science, vol. 42, no. 8, pp. 1093-1104, 1996.

[20] P. K. Banerjee and T. R. Turner, "A flexible model for the pricing of perishable assets," Omega, vol. 40, no. 5, pp. 533-540, 2012.

[21] A. Rajan and S. R. Rakesh, "Dynamic pricing and ordering decisions by a monopolist," Management Science, vol. 38, no. 2, pp. 240-262, 1992.

[22] R. V. Ramasesh, "Lot-sizing decisions under limited-time price incentives: a review," Omega, vol. 38, no. 3-4, pp. 118-135, 2010.

[23] Y. Levin, J. McGill, and M. Nediak, "Dynamic pricing in the presence of strategic consumers and oligopolistic competition," Management Science, vol. 55, no. 1, pp. 32-46, 2009.

[24] C. H. Lee, T. M. Choi, and T. Cheng, "Selling to strategic and loss-averse consumers: stocking, procurement, and product design policies," Naval Research Logistics (NRL), vol. 62, no. 6, pp. 435-453, 2015.

[25] O. Perdikaki and J. Swaminathan, "Improving valuation under consumer search: implications for pricing and profits," Production and Operations Management, vol. 22, no. 4, pp. 857874, 2013.

[26] M. Yu, R. Kapuscinski, and H. Ahn, "Advance selling: effects of interdependent consumer valuations and seller's capacity," Management Science, vol. 61, no. 9, pp. 2100-2117, 2015.

[27] Y. Aviv and A. Pazgal, "Optimal pricing of seasonal products in the presence of forward-looking consumers," Manufacturing \& Service Operations Management, vol. 10, no. 3, pp. 339-359, 2008.

[28] E. Adida and O. Ozer, "Why markdown as a pricing modality?," Management Science, vol. 65, no. 5, pp. 2161-2178, 2019.

[29] G. Gallego, R. Phillips, and Ö. Şahin, "Strategic management of distressed inventory," Production and Operations Management, vol. 17, no. 4, pp. 402-415, 2010.

[30] G. P. Cachon and P. Feldman, "Price commitments with strategic consumers: why it can be optimal to discount more frequently... than optimal," Manufacturing \& Service Operations Management, vol. 17, no. 3, pp. 399-410, 2015.

[31] Ö. Özer and Y. Zheng, "Markdown or everyday low price? The role of behavioral motives," Management Science, vol. 62, no. 2, pp. 326-346, 2015.

[32] T. Choi and C. Chiu, Eds., Risk Analysis in Stochastic Supply Chains: A Mean-Risk Approach, Springer Science \& Business Media, New York, 2012.

[33] Z. Wu, W. Zhu, and P. Crama, "The newsvendor problem with advertising revenue," Manufacturing \& Service Operations Management, vol. 13, no. 3, pp. 281-296, 2011.

[34] G. P. Cachon and R. Swinney, "The value of fast fashion: quick response, enhanced design, and strategic consumer behavior," Management Science, vol. 57, no. 4, pp. 778-795, 2011.

[35] M. S. Chen and C. C. Chuang, "An extended newsboy problem with shortage-level constraints," International Journal of Production Economics, vol. 67, no. 3, pp. 269-277, 2000.

[36] Y. Li, F. Ye, and Q. Lin, "Optimal lead time policy for short life cycle products under conditional value-at-risk criterion," Computers and Industrial Engineering, vol. 88, no. 88, pp. 354-365, 2015.

[37] Y. Qin, J. Wang, and C. Wei, "Joint pricing and inventory control for fresh produce and foods with quality and physical quantity deteriorating simultaneously," International Journal of Production Economics, vol. 152, pp. 42-48, 2014.

[38] Y. Qin, R. Wang, A. J. Vakharia, Y. Chen, and M. M. H. Seref, "The newsvendor problem: review and directions for future research," European Journal of Operational Research, vol. 213, no. 2, pp. 361-374, 2011.

[39] J. Wang, L. Wang, F. Ye, X. Xu, and J. Yu, "Order decision making based on different statement strategies under stochastic market demand," Journal of Systems Science and Systems Engineering, vol. 22, no. 2, pp. 171-190, 2013.

[40] J. Wang, S. Zhang, C. Wu, J. Yu, C. Chen, and S. Tsai, “Timesensitive markdown strategies for perishable products based on dynamic quality evaluation," Kybernetes, vol. 3, no. 7, pp. 1-16, 2020

[41] P. S. Taoukis and T. P. Labuza, "Applicability of timetemperature indicators as shelf life monitors of food products," Journal of Food Science, vol. 54, no. 4, pp. 783-788, 1989.

[42] G. Fibich, A. Gavious, and O. Lowengart, "Explicit solutions of optimization models and differential games with nonsmooth (asymmetric) reference-price effects," Operations Research, vol. 51, no. 5, pp. 721-734, 2003.

[43] R. Sayner, "Markups \& markdowns," Independent Retailer, p. 1, 2011.

[44] G. Gallego and G. Van Ryzin, "Optimal dynamic pricing of inventories with stochastic demand over finite horizons," Management Science, vol. 40, no. 8, pp. 999-1020, 1994.

[45] G. Khatwani and P. R. Srivastava, "Impact of information technology on information search channel selection for consumers," Journal of Organizational and End User Computing, vol. 30, no. 3, pp. 63-80, 2018.

[46] N. Suyel and R. Pinki, "PpBAC: popularity based access control model for cloud computing," Journal of Organizational \& End User Computing, vol. 30, no. 4, pp. 14-31, 2018.

[47] L. Fabisiak, "Web service usability analysis based on user preferences," Journal of Organizational and End User Computing (JOEUC), vol. 30, no. 4, pp. 1-13, 2018.

[48] J. Jakli, T. Grubljei, and P. S. Coelho, "The shift to socioorganizational drivers of business intelligence and analytics acceptance," Journal of Organizational and End User Computing (JOEUC), vol. 31, no. 2, pp. 37-64, 2017.

[49] A. Shahri, M. Hosseini, K. Phalp, J. Taylor, and R. Ali, "How to engineer gamification: the consensus, the best practice and the grey areas," Journal of Organizational and End User Computing, vol. 31, no. 1, pp. 39-60, 2019. 